\title{
Les Cités Maudites Selon le Coran : Un Rude Châtiment
}

\author{
Majida Sayegh, \\ Université Libanaise, Facultés des Lettres et des Sciences Humaines \\ (Section 5) Département de Langue et Littérature Françaises
}

Doi:10.19044/esj.2020.v16n20p78 ～URL:http://dx.doi.org/10.19044/esj.2020.v16n20p78

\section{Résumé}

Cet article étudie profondément les différents types de l'iniquité dans les cités maudites selon le texte coranique : idolâtrie, ignorance, corruption, immobilisme et turpitude. Les Envoyés qui visaient la reforme suivirent un long processus d'argumentations et de preuves logiques en validant un principe selon lequel la corruption conduit à la destruction. Le principe de « la bonne mesure et le bon poids » conduit à la justice et à la prospérité. Accumuler les reformes aboutit à l'évolution des sociétés humaines. Mais, les Envoyés n'avaient récolté, de la part de leurs peuples, que mépris, insultes et menaces. L'insistance des cités à leurs multiples corruptions rendait la reforme impossible, ce qui aboutit à l'intervention divine selon laquelle la preuve précède toujours le châtiment. La destruction prenait de diverses formes : déluge, vent mugissant, cri ou pierres d'argile. Actuellement, les sites archéologiques d'Al-Hijr en Arabie saoudite, et de Tall El-Hamam en Jordanie témoignent respectivement et incontestablement de (Madā'in Șāliḥ) et de la cité de Sodome.

Mots-clés: Iniquité, multiples corruptions, Messager, raison, preuves logiques, reforme, châtiment, destruction 


\title{
The Cursed Cities according to the Qur'an: A Cruel Punishment
}

\author{
Majida Sayegh, \\ Université Libanaise, Facultés des Lettres et des Sciences Humaines \\ (Section 5) Département de Langue et Littérature Françaises
}

\begin{abstract}
This article examines in depth the different types of iniquity in the accursed cities according to the Koranic text: idolatry, ignorance, corruption, immobility, and turpitude. A reform was embarked upon by Messengers who followed a long process of arguments and logical evidence in validating a principle that corruption leads to destruction. The principle of "the right measure and the right weight" leads to justice and prosperity. Accumulating reforms leads to the evolution of human societies. The Messengers, however, received contempt, insults, and threats from their people. The insistence of the cities on their multiple corruptions made the reform impossible, which led to the divine intervention that the proof always precedes the punishment. Destruction took various forms such as flood, howling wind, scream, or clay stones. Currently, the archaeological sites of Al-Hijr in Saudi Arabia and Tall el-Hamam in Jordan, respectively, gives an unquestionably evidence of Madā' in Șālih and the city of Sodom.
\end{abstract}

Keywords: Iniquity, multiple corruptions, Messenger, reason, logical proofs, reform, punishment, destruction

\section{Introduction}

Le texte coranique fascine pour de multiples raisons : le symbolisme de son langage, sa concision, et surtout son incantation qui rythme les idées les plus abstraites mais aussi les récits les plus captivants. Comme le dit (Gouguenheim, 2008, p.136-137) : «Par sa structure, la langue arabe se prête en effet magnifiquement à la poésie. Chaque mot y est composé à partir d'une racine de trois consonnes, que l'on peut compléter à l'aide d'autres consonnes, et de trois voyelles. Ce système facilite les répétitions de sons, procure des effets d'harmoniques, amplifiés par le rythme que produit un système consonantique de fortes et de faibles ». Aussi, dans ce contexte, Le Clézio, Prix Nobel de la littérature 2008, est séduit par cette «langue-musique [...] qui s'enroule autour de toi et va jusqu'à ton cœur» (Barbey et al., 2003, 
p.138). Effectivement, la langue arabe se caractérise par sa musicalité mais aussi par la richesse de son vocabulaire qui permet d'ouvrir un large spectre d'investigation et de compréhension. C'est pourquoi, il y a plus de 100 interprétations du Coran selon l'Organisation Islamique pour l'Education, les Sciences et la Culture (ISESCO). Selon une étude statistique sur les verbes, les racines et les structures dans la langue arabe, faite par (Alam, 2016), le Coran comporte 1767 racines et 77476 mots. En effet, la langue arabe contient 7420 racines de verbes composées de trois ou quatre caractères sans nier la présence des structures binaires et des racines de verbes de cinq caractères. A ce propos, il serait utile de donner la définition du mot « racine ». Selon (Abd Al Haye, 2016, p.65) « la racine linguistique désigne les entrées linguistiques dépouillées des extensions », elle est un élément de base du mot, dépourvu de tout affixe. Cette racine représente donc des lettres communes parmi un grand nombre de mots qui sont dérivées les unes des autres. Cela donne à chaque vocabulaire des différentes dimensions individuelles en fonction de leur utilisation dans le texte.

Réellement, le texte coranique a occupé une place importante dans les études théologiques, philologiques, linguistiques ou même historiques. Selon (De Prémare, 2006) un historien et un universitaire français, spécialiste de la langue et de la culture arabes et de l'histoire de l'Islam, « le Coran ne raconte pas donc ni la naissance d'une nouvelle religion, ni la vie de Muhammad, ni la conquête arabe, ce n'est pas un livre d'histoire, un récit historique, c'est autre chose ». C'est dans cette « autre chose » que cette recherche tente d'explorer le monde des cités maudites. Le présent article est guidé par deux principes: le respect rigoureux du texte littéral du Coran et l'approche thématique du sujet. En réalité, étudier séparément les versets coraniques les prive d'une grande partie de leur lisibilité. Dans ce contexte, (Frégosi, 2002) voit que durant la période médinoise (622-632) de l'apostolat ( $a l$ da'wa) du Muhammad, le message coranique a pris une tendance à traiter des thèmes liés à l'organisation de la vie dans la cité de Médine. Il parle de « soixantaine de versets dits juridiques, soixante-dix versets en matière commerciale, trentaine de versets relatifs aux châtiments, et seule une dizaine de versets peut également être qualifiée de politiques ».

Il est primordial de signaler que le Coran raconte l'histoire de certains Messagers, et pour d'autres ne fait que mentionner les noms sans apporter aucune autre information à leur sujet (Clément, 78) «Avant toi, déjà, Nous avons envoyé des Messagers. Il en est parmi eux dont Nous t'avons raconté l'histoire, et d'autres dont Nous ne t'avons rien raconté... ». D'un point de vue terminologique, il n'y a pas de différence entre un Prophète et un Messager d'après (Fadlallah, 1998, p.356). Selon la statistique, en particulier la théorie de l'échantillonnage, il est incontestable que dans toutes les enquêtes quantitatives, la taille de l'échantillon est un facteur clé pour obtenir des 
données fiables. Du point de vue statistique, la taille de l'échantillon associé aux 25 Messagers mentionnés dans le Coran, reflète adéquatement l'essence de leur message divin adressé à l'humanité, non seulement en termes de nombres mais aussi en termes de savoir rationnel et moral. Pour plus d'informations sur la taille de l'échantillon, on peut consulter l'ouvrage de (Richard \& Gouri, 1996). Par ailleurs, le manque d'études qui insistent sur les thèmes économiques et sociaux dans le Coran incite à rechercher dans ce domaine sans entrer dans les méandres du confessionnalisme au sein de l'Islam, ni dans les divergences qui pourraient exister entre les exégètes.

Cette lecture objective des versets essaie d'examiner le système d'idées qui reflètent les concepts et les comportements qui prévalaient dans ces temps anciens afin de déterminer les formes de corruption qui régnaient et de comprendre le contenu des appels des Messagers. Réellement, deux parties sont en conflit : la première correspond à la catégorie encroûtée et intolérante qui s'efforce de perpétuer l'immobilisme sur les plans moral et matériel, et la seconde est représentée par les Messagers et le peu des gens qui les avaient suivis en essayant de libérer leurs cités de la corruption, de l'injustice et de l'oppression.

Concernant la méthodologie suivie dans cet article, il est important de fixer les points suivants :

1. Le texte coranique, à travers son contenu informatif, était traité comme tout texte littéraire sans se focaliser sur sa dimension sacrée.

2. Le but de cette recherche n'est pas de prouver la véracité ou l'historicité des récits coraniques : le sujet est purement thématique visant à faire ressortir tout simplement la vision coranique les maux dont souffraient les cités maudites en insistant sur les facteurs économiques et sociaux.

3. Les analyses se basent sur la traduction française du texte coranique et non pas sur la version originelle en langue arabe. Les allusions aux diverses significations de certains termes de la langue arabe ont pour but d'expliciter davantage la visée du Coran, ce qui va dans le sens de l'étude : analyser le contenu informatif des versets.

4. L'intérêt se porte donc sur la littéralité du mot et non sur son aspect poétique. En effet, un mot est « un être, une entité et que probablement il n'y a pas de synonymes, ..., C'est pour ça que je crois que c'est impossible de traduire la poésie » comme le dit Borges lors d'un entretien (Bell, 1989).

5. Les explications relatives à la langue arabe voulaient faire allusion à certaines de ses caractéristiques afin que le lecteur puisse prendre une idée de la langue avec laquelle le Coran était écrit à l'origine.

6. Les références des certaines découvertes archéologiques et des études scientifiques récentes témoignent de l'existence historique de ces cités. 
Pourquoi les notables opulents n'avaient pas répondu positivement aux Messagers ? Est- ce l'immobilisme inhérent à leur être moral ? Est-ce leur intérêt à conserver le contrôle de la société avec ses fondements religieux et économiques ? Est-ce le refus de tout caractère ouvert au dialogue et le maintien d'un esprit fermé et répressif guidé par l'arrogance et le désir de la domination ? Est-ce l'attrait des plaisirs qui faisait endormir la raison ? Est-ce la vanité qui a poussé les cités injustes à ne pas écouter les appels des réformistes ? Et par ce fait sont-elles (Al-Talâq, 8-9) "goûté les fruits amers de leur conduite, et leur comportement a fini par causer leur perte». Réellement, le Coran contient 57 versets dont chacun contient le mot (alqarīä̈ : la cité), 50 versets qui mentionnent (al-fasād : la corruption) et ses extensions. Le corpus est tiré de la version française du Coran selon le site (Electronic Qur'an, www.E-Quran.com, 2009) qui contient des traductions en 58 langues différentes.

Cette étude est divisée en cinq sections : la présente introduction constitue la première tandis que la deuxième sera consacrée à une exploration complète de la réalité des cités maudites figurant dans le texte coranique en suivant les repères de l'iniquité, ce qui va permettre de faire un constat de la réalité de la corruption. La troisième est consacrée aux réactions des cités face aux Messagers. La quatrième étudie les trajets respectifs des Envoyés, selon un ordre chronologique, Noé, Houd, Sâlih, Loth et Chu'aïb dont les cités ont été signalées maudites. Enfin la conclusion constituera la cinquième et dernière section.

\section{Lecture approfondie de l'iniquité dans les cités maudites}

Cette section est consacrée aux versets (āyāt) dans lesquels le terme « la cité : al-qarīä̈ » a été mentionné afin de connaitre les concepts religieux et économiques qui ont été prévalus à l'époque, ainsi que leurs conséquences comportementales sur les groupes sociaux, ce qui révèle une idée claire des relations établies, d'une part, entre les individus et leurs supérieurs, et d'autre part, la société et l'héritage des anciens.

Avant tout, il serait utile de saisir les sens de certains mots qui seront utilisés dans cette recherche en adoptant des sens utilisés par (Déclais, 2015) : aï : Il ne s'agit pas toujours d'un verset, mais il peut s'agir d'un étendard, signe de ralliement, puis une chose étonnante, prodige de la nature ou événement miraculeux.

Qaūm : Ce n'est pas toujours un «peuple » au sens ethnique du terme. Il peut s'agir d'une communauté d'origine ou d'opinion.

Le Coran révèle que l'iniquité de toutes sortes avait été prévalue dans les cités maudites. Effectivement, les oppresseurs représentés par les notables orgueilleux et méprisants régnaient en force dans tous les secteurs de la vie communautaire. Avant un examen approfondi des versets associés aux cités, 
il est nécessaire de passer rapidement sur ce que le mot « iniquité » (al-zwulm) signifie dans la langue arabe : le mot («al-zwulm») et ses dérivés, dont le mot « iniques »(al-zwalmin $)$ est le plus fréquent, apparaissent trois cent quinze fois dans le Coran. Cela désigne l'acuité de l'injustice qui régnait sur les individus, les groupes et les communautés.

Linguistiquement, selon le Lisān al- 'Arab, le verbe «zwalama » a un sens très concret : mettre une chose à une place qui n'est pas la sienne, c'està-dire «se tromper ». Ceci est pleinement reflété dans un proverbe arabe : « Celui qui place un loup comme berger se trompe « zwalama».

Un « mazlūm » est un opprimé, tandis que «al-ẓwālim » est celui qui commet l'iniquité. Donc, il ne s'agit pas d'une simple erreur faite involontairement mais c'est une mauvaise action moralement répréhensible. Il est remarquable que «l'obscurantisme » (al-z̧walāmīwä) est une doctrine qui ne favorise pas l'éducation du public (pour plus d'information sur l'obscurantisme dans les sciences humaines et sociales, voir (Elster, 2010). Le coran va plus loin dans le sens de «ténèbres » (al-z̧wulumāt), en soulignant qu'elles sont le contraire de la lumière : (Créateur, 19-20) «L'aveugle n'est pas pareil à celui qui voit », "ni les ténèbres à la lumière ». Il lie la foi à la clarté (La Vache, 257) "Dieu est le Protecteur de ceux qui croient: Il les fait sortir des ténèbres vers la lumière. Et ceux qui mécroient ont pour patrons les Tâghout, qui les font sortir de la lumière vers les ténèbres... ». Ainsi, comme l'obscurité (al-zwwul’mä̈) empêche la vision, l'injustice reflète l'aveuglement de l'esprit. Qu'elle soit commise par l'homme, intentionnellement ou involontairement, cela n'enlève rien à ses graves conséquences sur les sociétés.

Dieu s'est mis, Lui-même, à l'écart de l'iniquité comme le prouvent de nombreux versets, par exemple (Les Femmes, 40) «Dieu ne lèse personne, fût-ce du poids d'un atome ... », (La Famille De Imrân, 108) " ... Et Dieu ne veut pas d'iniquité pour les mondes », (La Caverne, 49) «...Ton Seigneur ne lèsera personne », (Clément, 31) " ... Pourtant, Dieu ne veut pas d'iniquité envers Ses serviteurs!»

L'iniquité qui était en tout temps et en tout lieu, est actuellement combattue par toutes les législations civiles qui incitent à l'obligation de sa prévention. Ce thème est un vaste domaine dans le texte coranique, c'est pourquoi, la présente recherche traite uniquement les types d'iniquité qui s'étaient produits dans les cités maudites :

- L'iniquité due à l'idolâtrie.

- L'iniquité due aux notables opulents (al-mutrafinn) .

- L'iniquité due à l'immobilisme (al-ğumūdīwä̈) .

- L'iniquité due aux turpitudes commises. 


\section{II.1 L'iniquité due à l'idolâtrie}

Selon le dictionnaire français (Larousse) : «L'idolâtrie est un culte rendu à des idoles ou à des créatures adorées comme la divinité même », autrement dit il s'agit d'une fausse divinité, une sorte d'affabulation et de parjure comme indique (Fadlallah, 1998, p.19, p.202). Selon le Coran, le polythéisme est l'un des plus grands types d'iniquité, c'est ce qu'il a recommandé Luqmân à son fils (Luqmân, 13) «" O mon fils! N'associe rien à Dieu. Lui donner des associés est une monstrueuse iniquité " : l'allégation de la divinité est un grand péché parce qu'elle résulte de la tyrannie et des mensonges flagrants. Le propos du Pharaon lors de son défi à Moïse en est un exemple (Ceux Qui Arrachent, 24) " ...disant : Je suis votre souverain suprême!», dans cette affirmation, il a commis un sacrilège qui a causé son châtiment ultérieur: (Ceux Qui Arrachent, 25) «Dieu lui infligea alors le tourment de l'Au-delà et de l'Ici-bas».

En suivant le mouvement des Messagers, l'attachement des cités maudites à l'idolâtrie était flagrant comme c'était le cas du peuple de Noé : (Noé, 23) «Et ils ont dit : "N'abandonnez pas vos divinités : n'abandonnez ni Wadd, ni Souwa', ni Yaghouth, ni Ya'ouq, ni Nasr !», et la répétition deux fois de la négation du verbe (abandonner) n'est qu'une insistance à la maintenir fermement.

De même, le prophète Houd avait appelé son peuple afin d'abandonner les idoles et de tourner vers Dieu (Al-A'râf, 70) «Ils dirent: Es-tu venu à nous pour que nous adorions Allâh, lui seul, et que nous délaissions ce que nos pères adoraient ... ? », (Houd, 53) «Ils dirent : O Houd! Tu ne nous as pas apporté une preuve évidente, et nous n'abandonnerons pas nos divinités sur ta parole... ». Cela s'était également produit avec le Prophète Sâlih (Houd, 61) «Aux Thamoud Nous envoyâmes leur frère Sâlih. Il dit : O mon peuple! Adorez Dieu! Vous n'avez d'autre dieu que Lui... ». Comme les autres les autres Messagers qui l'avaient devancé, Chu'aỉb avait été envoyé pour guider le peuple de Madyan vers le monothéisme (Al-Hijr, 79) «Les habitants d'AlAïka ("la Forêt" de Madyan) étaient pétris d'iniquité», (Houd, 84) "Aux gens de Madyan, Nous avons envoyé leur frère Chu'aïb. Il dit : O mon peuple! Adorez Dieu! Il n'y a pour vous d'autre dieu que Lui ! ... ». Mais ils l'avaient traité d'imposteur (Les Poètes, 176) «Les habitants d'al-Aikka ... Ont traité les envoyés d'imposteurs ». Pourtant, la question de l'idolâtrie n'est pas évoquée directement au sujet du peuple de Loth qui avaient traité (Les Poètes, $160)$ "les envoyés d'imposteurs » mais l'accent est mis sur leurs turpitudes morales et leur malhonnêteté (Les Prophètes, 74) «...Nous l'avons sauvé de la cité qui se livrait aux turpitudes. Ces gens-là étaient malfaisants et pervers $»$. 


\section{II.2 L'iniquité due aux habitants opulents (al-mutr)fin)}

Le terme (al-mutrafin) est le pluriel de (mutraf), une personne opulente qui « fait ce qu'elle désire et que personne ne peut l'en empêcher» comme la définit (Fadlallah, 1998, p.14, p.68). C'est donc jouir insolemment de son abondance en se livrant, sans retenue, aux plaisirs et à la luxure de toutes sortes.

D'après les versets coraniques, le rôle des hommes riches est déterminant dans le sort malheureux des cités condamnées car ils pratiquaient la corruption en soumettant le reste de leur communauté. Leurs seules lois étaient leurs propres intérêts : (Houd, 116) " ...Les iniques ont préféré suivre leur penchant pour la vie facile et ils se sont rendus coupables».

La référence à cette catégorie des gens fut à l'occasion de l'annonce de la naissance d'un enfant à Abraham qui a reçu des anges sous forme humaine (Ceux Qui Éparpillent, 32) «Ils dirent (par référence aux nobles hôtes d'Abraham) : Nous sommes envoyés vers un peuple criminel ». Le verset parle du peuple de Loth célèbre par sa débauche et sa méchanceté. Dirigeants de leurs cités, les criminels ou (al-mujrimun) se caractérisent par la transgression et la perversité (Le Bétail, 123) "Dans chaque cité, Nous avons placé des grands criminels pour qu'ils ourdissent des complots ... ». Par leur position sociale ils imposent leurs conditions au reste de leur communauté afin de préserver leur pouvoir économique et social.

Mais comme confirme (Fadlallah, 1998, p.9, p.314) «Le crime ne reste pas chez ses fabricants, il s'étend à toute la communauté, en particulier lorsque la tromperie et l'escroquerie se sont transformées en un flux social pour se terminer par l'agression de personnes ». La corruption se propage dans la cité en dominant les diverses articulations de la vie quotidienne, et l'injustice devient excessive ouvrant ainsi la voie à la destruction. Les opulents propageaient des idéologies criminelles afin de maintenir l'ignorance et renforcer leur pouvoir. Selon (Fadlallah, 1998, p.14, p.51) «Lorsque les habitants opulents détiennent le pouvoir, ils exercent l'abus d'autorité, ils propagent la violence au nom de leur idéologie dépassée, de telle manière la corruption règne sur tous les plans économiques et sociaux».

Autre stratégie de ces dirigeants était de consolider les anciennes croyances sous prétexte de préserver l'héritage des anciens (Les Poètes, 74) « Ils dirent : Non! mais nous avons trouvé nos pères qui agissaient de la sorte », (Les Ornements D'or, 23) «... Nous n'avons envoyé aucun avertisseur dans une cité sans que ceux qui y prospéraient ne disent: Oui, nous avons trouvé nos pères suivant tous une même voie, et nous marchons sur leurs traces». Ces habitants opulents étaient visés par tout appel à la reforme selon le Coran (Le Voyage Nocturne, 16) «Lorsque Nous avons voulu détruire une cité, Nous avons d'abord lancé un ordre [d'obéissance] à ses habitants opulents, mais ils $s^{\prime} y$ sont livrés à des perversités ... ». Et cette catégorie devient un obstacle 
majeur à toute réforme (Saba, 34) «Jamais Nous n'avons envoyé à une cité un avertisseur sans que les citoyens aisés déclarent : Nous ne croyons pas à votre message !». Ainsi, l'aisance matérielle des opulents approfondissait le fossé avec les Messagers et s'affirmait comme source du mal : la stagnation de la société garantissait la continuité de leurs privilèges. Ils se voyaient comme les seuls dignes d'exprimer leurs opinions et leurs désirs tandis les humbles du reste de la société n'en avaient pas le droit. C'est ainsi que les notables criminels du peuple de Noé l'avaient fait valoir (Houd, 27) « ... Nous ne voyons que les plus méprisables d'entre nous qui t'aient suivi au premier coup d'œil ... ", (Les Poètes, 111) "Ils dirent: "Croirons-nous en toi alors que ce sont les hommes les plus méprisables qui te suivent? ». Selon ces riches qui (Les Croyants, 33) « ...niaient la rencontre de la vie future... » l'aisance matérielle était le seul critère de valorisation.

\section{II.3 L'iniquité due à l'immobilisme (al-ğumūdīwä̈)}

L'iniquité est traitée ici comme l'une des conséquences de l'immobilisme des idées. Ce n'était pas l'apanage d'une minorité de fortunés mais un cas généralisé qui sévissait dans la mentalité collective. Dans ce contexte, (Shahrur, 2018) écrit que «Chaque société gouvernée par un esprit arriéré et déplacé porte les germes de sa propre destruction et de son propre tourment [...] L'iniquité ne peut se propager dans une cité que si le comportement de la majorité est une perversion attestée ».

Dirigés par leurs chefs aisés, les masses n'avaient aucun argument rationnel basé sur la raison pour s'opposer aux Messagers. L'attrait de l'héritage ancien était trop ancré dans les mentalités (La Vache, 170) « Lorsqu'on leur dit : Suivez ce que Dieu a révélé, ils répondent : Non, nous suivons la coutume de nos pères ! », (Al-Mâ'idah, 104) «Lorsqu'on leur dit :... venez à l'Envoyé ! Ils répondent : Ce que nous avons trouvé chez nos pères nous suffit. Et quoi donc si leurs pères ne savaient rien et s'ils n'étaient pas dirigés ?». Suivre les coutumes et les croyances des ancêtres à l'aveuglette était la seule règle.

La question d'association avec Dieu était une extension naturelle de l'héritage des parents, et donc suivre leurs traces était une obligation pratiquée automatiquement : (Al-A'râf, 173) « ... Avant nous, nos pères avaient pris des associés, et nous sommes leur postérité... ». L'un des faux arguments des peuples des cités maudites pour justifier leur pratique était (Le Bétail, 148) « Si Dieu l'avait voulu, ni nous ni nos pères n'aurions associé [quoi que ce soit à Dieu], et nous n'aurions déclaré aucun interdit ... ». Ces faux arguments étaient la base de leurs ripostes aux appels des Messagers. Ils considéraient l'adoration des idoles comme une fatalité et ripostaient violemment aux Messagers : les ('Ad) disaient à Houd (Al-A'râf, 70) « Es-tu venu à nous pour que nous adorions Allâh, lui seul, et que nous délaissions ce que nos pères 
adoraient ?», ainsi ce que Thamoud avait répliqué à Sâlih (Houd, 62) «... O Sâlih ! Tu étais, auparavant, un espoir pour nous. Veux-tu nous interdire d'adorer ce que nos pères adoraient ?... ». Pareillement, ce que les habitants d'Al-Aïka avaient rétorqué à Chu'aïb (Houd, 87) «... O Chu'aïb, ta façon de prier t'ordonne-t-elle que nous abandonnions ce que nos pères adoraient... ». Pour eux, les Envoyés n'étaient que de simples (Abraham, 10) «mortels» comme eux qui n'avaient pas le droit de les détourner des croyances de leurs ancêtres (Luqmân, 21).

\section{II.4 L'iniquité due à la turpitude morale.}

Selon le Coran, certaines cités ont été qualifiées de maudites à cause de leurs déviations du droit chemin. Pour cette raison, les Messagers étaient venus pour combattre les forces de la corruption qui asservissait les sociétés. Leurs buts étaient de préserver la valeur de l'être humain comme une créature pensante et valorisée par les facultés de raisonner et de méditer : ceci est signalé par les deux versets (Sad, 71,72) «Il advint donc que ton Seigneur dit aux anges : Oui, je vais créer d'argile un mortel », et "Lorsque Je l'aurai harmonieusement formé et que J'aurai insufflé en lui de Mon Esprit, tombez. prosternés devant lui ». Dans ce contexte, les cités maudites s'opposaient à cette perfection continuelle de l'homme en voulant l'enfermer dans une soumission complète à des croyances périmées et à une classe opulente qui en profitait afin de préserver ses privilèges.

Dans ce contexte, la criminalité du peuple de Loth était exemplaire dans sa corruption et sa mauvaise conduite: (L'araignée, 29) «Vous vous approchez des hommes, vous coupez les chemins (pour commettre du pillage) et vous vous livrez dans vos assemblées à des actions détestables... ". C'était la cité qui (Les Prophètes ,74) «se livrait aux turpitudes. Ces gens-là étaient malfaisants et pervers ». Ils se caractérisaient par leur insolence et leurs actions abominables auxquelles ils s'habituaient et s'accrochaient (Les Fourmis, 54) «... Vous livrez-vous à la turpitude en toute connaissance de cause?». Leurs forfaits étaient aggravés par leur incrédulité et leur insolence qui les avaient poussés à s'attaquer aux hôtes de Loth (anges sous forme humaine). L'agression était inhérente à leur personnalité, ils commettaient les turpitudes en toute liberté. C'est pourquoi les hôtes de Loth qui l'avaient averti du châtiment imminent de son peuple (Al-Hijr, 58) "Ils dirent : Nous sommes envoyés à un peuple de scélérats », (L’araignée ,31) « ... Certes, nous allons anéantir les habitants de cette cité. Ce sont vraiment des iniques ». En effet, ce peuple dépravé ne reculait devant rien et se moquait de Loth et de ceux qui étaient avec lui (Les Fourmis, 56) "Leur seule réponse fut de dire : Chassez. de votre cité la famille de Loth : ce sont des gens qui affectent la pureté». 


\section{Les accusations et les réactions contre les Envoyés}

Afin de renforcer leur autorité et leur influence dans tous les domaines, les opulents des cités maudites qui (Les Versets Clairement Exposé, 15) « se sont injustement enorgueillis sur la terre " s'appliquaient à inciter leurs peuples contre la figure du Messager : Houd en est un exemple (Les Croyants , 33) «Les notables de son peuple qui mécroyaient et niaient la rencontre de la vie future, et que Nous avions traités avec largesse dans la vie de ce monde, dirent : Celui-ci n'est rien d'autre qu'un simple mortel comme vous, il mange de ce vous mangez et boit de ce que vous buvez». Ainsi, (Abraham, 13) «Les mécréants dirent à leurs envoyés : Nous vous chasserons de notre pays, à moins que vous ne reveniez à notre religion ». C'était la seule réponse que les Envoyés recevaient des cités mécréantes. Dans ce contexte, il est important d'évoquer Abraham qui constitue l'archétype du prophète monothéiste maltraité par son peuple.

Le père des prophètes a été menacé d'être lapidé par son père qui l'avait ordonné de s'écarter de lui et de ses idoles (Marie, 46) «O Abraham, ... Si tu ne cesses pas, je te lapiderai. Éloignes-toi de moi pour un temps». Noé, aussi, endurait le même traitement de son peuple qui s'opposait à lui en le menaçant de lapidation (Les Poètes, 116) «Ils dirent : Si tu ne cesses pas, ô Noé, tu seras lapidé ». C'est ce qui était arrivé à Loth quand il s'était opposé à la perversion des habitants de sa cité, déclarant son horreur de leurs actions après les avoir avertis de leurs conséquences (Les Poètes, 167) «Ils dirent : Si tu ne cesses pas de nous importuner, ô Loth, tu seras banni !». La menace d'expulsion visait aussi les partisans de Loth (Al-A'râf, 82) « ... Chassez-les de votre cité ; ce sont des gens qui affectent la pureté ». Chu'aïb n'échappait pas à la règle : après avoir blâmé leur doctrine païenne et leur fraude dans les mesures et le poids (Al-A'râf, 88) "Ceux des notables de son peuple qui étaient orgueilleux dirent : Nous te chasserons de notre cité, ô Chu'aïb, toi et ceux qui ont cru avec toi, à moins que vous ne reveniez à notre religion... ». Il est important de souligner que l'interjection (Ô) (dans « ô Noé », « ô Loth », «ô Chu'aïb ») reflète un sentiment exalté de refus de la part de ces peuples confirmant leur détermination à bannir les Messagers qui représentaient pour eux une menace de leur stabilité culturelle et matérielle. Pour cela, ils les menaçaient de mort ou de lapidation s'ils ne renonçaient pas à leurs idées réformatrices.

Pour les habitants de ces cités, il n'y avait pas de compromis avec les Envoyés. Il s'agissait vraiment de deux types de tendances sociales qui s'opposeraient : d'une part, un courant de réforme ouvert et guidé par une vision réfléchie et globale du destin de l'homme, et d'autre part, un bloc stagnant dirigé par une classe opulente qui faisait régner ses croyances rétrogrades et ses idées erronées. En réalité, les réactions des notables des cités maudites contre la personne du Messager étaient arrogantes et violentes : 
leur but était de le défigurer pour le rabaisser aux yeux du peuple : (Al-A'râf, 160) «Nous te voyons dans un égarement manifeste ». (Houd, 38) " ... et chaque fois que des notables de son peuple passaient près de lui, ils se moquaient de lui... ». Les notables n'avaient pas d'arguments contre lui que de l'accuser de fou (Les Croyants, 25) «Il n'est qu'un homme possédé par les djinns... ». Pour eux, ils ne pouvaient pas suivre un homme égaré car son appel à adorer Dieu et rejeter l'idolâtrie était une atteinte à leur foi, et d'après leurs concepts sociaux, seul un fou aurait prêché ce genre de fait. Il semble, que par humiliation, ils avaient demandé à Noé d'expulser les pauvres qui croyaient en lui, mais Noé leur répondait (Les Poètes, 114) «Ce n'est pas moi qui repousserai les croyants ».

Quant aux ('Ad), ils ont annoncé clairement qu'ils ne croyaient pas en Houd (Al-A'râf, 76) «Les orgueilleux reprirent : Ce à quoi vous croyez, nous le rejetons! ». Ils l'avaient accusé d'être étourdi et calomniateur (Al-A'râf, 66) "Les notables de son peuple qui mécroyaient dirent : Nous te voyons plongé dans la folie et nous pensons même que tu es un menteur! ». Ils avaient affiché leur puissance et leur fierté face à lui. Leur arrogance et leur entêtement étaient excessifs en déclarant qu'ils étaient indifférents à tout appel (Les Poètes, 136) " ... Il nous est indifférent que tu nous exhortes, ou que tu ne nous exhortes pas ». Ils s'accrochaient à leurs ancêtres en tant que modèles à suivre: (Les Poètes, 137) «Tout cela se sont les choses auxquelles les Anciens croyaient déjà ». Selon eux, la vie se termine par la mort, et il n'y a pas de résurrection (Les Croyants, 35,36) «Vous promet-il qu'une fois morts, lorsque vous serez poussière et ossements, on vous fera resurgir?», et «Foin, foin des promesses qui vous sont faites! ». L'utilisation du mot "foin" (haīhāt) deux fois n'est qu'une affirmation de leur mépris pour le jour de la Résurrection. En fin de compte, ils étaient arrivés à une conclusion selon laquelle l'obéissance à Houd est une perte à tous les niveaux (Les Croyants, 34) «Or, si vous obéissez à un simple mortel comme vous, vous serez. certainement parmi les perdants ».

Les gens de Sâlih l'accusaient aussi d'être un simple mortel comme eux (Les Poètes, 154) «Tu n'es qu'un mortel comme nous. Apporte-nous donc un signe, si tu es véridique!». Alors comment pouvaient-ils suivre quelqu'un de mortel ? Pour eux, il n'était (Les Poètes, 153) « ...qu'un homme ensorcelé » et ceux qui le suivaient n'étaient que des (Al-A'râf, 75) «faibles » qui n'inspiraient que de l'aversion (Les Fourmis, 47). Leur arrogance les avait conduits à rejeter son message (Al-A'râf, 76) «Les orgueilleux reprirent: Ce à quoi vous croyez, nous le rejetons !». Ils jugeaient ses avances avec dérision en se demandant comment un homme comme lui pouvait être choisi comme messager (La Lune, 25) «Se peut-il que, parmi nous tous, ce soit sur lui que le rappel ait été lancé ? Non! C'est un imposteur, un insolent!». 
Les notables de Thamoud avaient préféré (Les Versets Clairement Exposés, 17) « ... l'aveuglement à la bonne direction ... ». Ils avaient déclaré en pleine voix leur rejet à l'appel de Sâlih et (Al-A'râf, 77) «Ils tranchèrent les jarrets de la chamelle, en rébellion contre l'ordre de leur Seigneur... ». Cette chamelle qui symbolisait l'équité divine à respecter.

Chu'aïb n'avait pas plus de chance avec les habitants de Madyan (Les Poètes, 176) «Les habitants d'al-Aika ... ont traité les envoyés d'imposteurs ». Malgré son éloquence, ils ne comprenaient pas ses sermons (Houd, 91) « Ils dirent: O Chu'aïb! Nous ne comprenons pas grand'chose à ce que tu dis. Nous voyons que tu n'as guère de prestige parmi nous... ». Ils détestaient ce qu'il disait et s'en détournaient en l'accusant de faiblesse.

Les notables de Madyan s'étaient comportés comme ceux de Sâlih : (Les Poètes, 185,186) «Ils dirent: "Tu n'es qu'un homme que l'on a ensorcelé », " et tu n'es qu'un mortel comme nous. Nous pensons que tu es un imposteur ». Leur arrogance arrivait jusqu'à lui demander de faire tomber un fragment du ciel sur eux comme une preuve de sa sincérité (Les Poètes, 187) «Fais donc tomber sur nous un fragment du ciel si tu es véridique!». Mais cette proclamation n'était qu'une assertion de l'ignorance dans laquelle ils étaient engloutis. En effet, le peuple de Madyan était caractérisé par la fraude dans les mesures et le poids. Les notables de cette cité se considéraient libres de leurs profits et de leur argent, c'est pourquoi leur réaction était violente contre Chu'aïb et ses disciples qui luttaient contre ce fléau social. Mais le secours de Dieu aux Messagers n'allait pas tarder comme cela est montré dans la section qui suit.

\section{Les Messagers de Dieu face aux cités maudites et leurs châtiments}

Les Messagers se montraient d'abord cléments avec leurs peuples en les invitant à la réforme, d'une part, pour éviter des représailles violentes de la part des grands criminels (akābiru al-muğrimīn') et d'autre part, pour laisser les gens réfléchir selon la raison.

\section{IV.1 Appel de Noé}

Noé se distingue par son ancienneté, sa persévérance et son exemplarité : avant Abraham, il représente une figure archétypale du Messager défié et rejeté au niveau terrestre. Par sa longue vie et sa lutte pour préserver les êtres vivants à l'aide de son arche, il représente l'acharnement de la foi et l'amour de la vie contre le mal et l'anéantissement des valeurs. D'ailleurs (Taharraoui, 2015) a identifié un scénario de la vie de Noé composé des 11 principaux éléments constitutifs qui s'étendent du début de la mission jusqu'au débarquement.

Noé qui aimait son peuple avait commencé par l'inviter à renoncer au paganisme en le prévenant d'un châtiment divin s'il ne prenait pas ses appels 
au sérieux (Al-A'râf, 59) «... Il dit : O mon peuple ! Adorez Dieu ! Il n'y a pas, pour vous, d'autre dieu que Lui. Certes, je crains pour vous le châtiment d'un Jour terrible ». Il se présentait comme l'un d'eux, un conseiller et un guide qui n'était pas venu de sa propre initiative mais qu'il était un Messager du Seigneur. Noé avait déclaré qu'il ne voulait en échange ni indemnité, ni gloire (Houd, 29) «O mon peuple! Je ne vous demande en échange aucune richesse. Ma récompense n'incombe qu'à Dieu... ». (Houd, 31) Il ne prétendait pas la connaissance du mystère, il ne se mettait pas aux rangs de rois. Avec cette déclaration éthique, Noé voulait transmettre un message d'humilité et de respect à son peuple. Aucune contrainte n'était exprimée dans son appel, sachant que la contrainte empêchait de voir la vérité (Houd, 28) «O mon peuple!... Si je m'appuie sur une preuve évidente venue de mon Seigneur et s'Il m'a gratifié d'une miséricorde qui vous est occultée, pensez-vous que nous puissions vous l'imposer contre votre gré?».

Il avait appelé son peuple à dialoguer en déclarant ce qu'il pensait en secret (Jonas, 71) « ... Mettez-vous donc d'accord sur votre dessein et rassemblez vos associés sans que votre affaire vienne vous tourmenter... ». (Houd, 27) «Les notables de son peuple, qui mécroyaient, dirent : Nous ne voyons en toi qu'un mortel semblable à nous... », (Les Croyants, 24) «... Qui donc est celui-ci... alors que si Dieu l'avait voulu, Il aurait fait descendre des anges... ». Selon leur reproche, Noé n'était pas un ange, c'était un homme, il n'était pas différent d'eux, par conséquent, ils n'étaient pas prêts à écouter ce qu'il prêchait. Les notables lui avaient demandé d'expulser les croyants pauvres considérés comme méprisables mais il leur avait rétorqué (Les Poètes, 114) «Ce n'est pas moi qui repousserai les croyants», (Houd, 29) «... mais je vois que vous êtes un peuple d'ignorants ».

La patience de Noé avec son peuple était exemplaire, ce qui est montré dans le verset (Noé, 5-9) :

(5) Il dit : "Mon Seigneur! J'ai appelé mon peuple nuit et jour,

(6) mais mon appel n'a fait qu'augmenter son éloignement,

(7) Chaque fois que je les ai appelés pour que Tu leur pardonnes, ils se sont mis les doigts dans les oreilles, ils se sont enveloppés dans leur vêtements, ils se sont obstinés et ont fait preuve d'un orgueil invétér,

(8) Je les ai ensuite appelés à voix haute,

(9) j'ai fait des proclamations et je leur ai parlé en secret.

Ces versets reflètent le travail acharné de Noé pour guider son peuple vers l'adoration de Dieu, ce qui n'avait fait que les exaspérer encore davantage (Houd, 32) «Ils dirent : O Noé! Tu as déjà disputé avec nous et tu as multiplié nos controverses. Fais donc venir ce dont tu nous menaces, si tu es véridique », ce qui explique combien de temps Noé avait passé avec son peuple 
(L’Araignée, 14) « .... Il demeura avec lui durant mille ans, moins cinquante ... ». Noé se désespérait, alors il appela son Seigneur pour l'aider (Les Croyants, 26) «Il dit : Mon Seigneur ! Prête-moi assistance contre leurs mensonges !», et Dieu lui a accordé le soutien (Les Rangs, 75) «Noé Nous a imploré, [Nous qui sommes] le Meilleur de ceux qui exaucent!», ce fût le terrible châtiment (L'Araignée, 14) " ... puis le déluge les emporta parce qu'ils étaient iniques », (Les Rangs, 76) «Nous l'avons sauvé, lui et sa famille, du terrible cataclysme ». (Les Prophètes, 77) «C'était un peuple malfaisant, et Nous les avons tous submergés ».

\section{IV.2 Appel de Houd}

Avant d'analyser l'appel de Houd à son peuple ('Ad), il est utile de mentionner qu'une étude scientifique récente faite par (GNOS et al., 2013) a eu lieu en Arabie saoudite dans la région 'Al-Hadidah.

Elle montre la présence du «matériau de sable de dune, lithifié contenant des structures sédimentaires parfaitement conservées, y compris des literies croisées et des terriers d'animaux ». Aussi, l'étude faite par (Prescott \& Robertson, 2004) a révélé une précision importante « dans la détermination de l'âge de cratères d'impact de météorite à Wabar (Al Hadida)» et les archéologues et les scientifiques espèrent déceler les mystères de cette cité.

Les ('AD) se caractérisaient par leur taille colossale et la solidité de leurs constructions dans le roc. Ce peuple de géants symbolisait l'arrogance titanesque de l'humain face au divin. C'est pourquoi, la mission de Houd n'était guère ordinaire. Malgré leur idolâtrie, il espérait l'adhésion de son peuple (Houd, 50) « ... O mon peuple! Adorez Dieu! Il n'y a pour vous d'autre dieu que Lui! Vous n'êtes que des affabulateurs!». En invitant les ('Ad) à adorer le Dieu Unique, Houd dénonçait en même temps leur vanité et leur folie de grandeur qui les poussait à construire de hauts lieux dénués de toute utilité rien que pour exposer leur force : (Les Poètes, 128) «Allez-vous construire sur chaque colline un monument pour vous divertir?». Assurément, ils se comportaient comme s'ils étaient éternels en bâtissant des châteaux impénétrables.

Leur travail était donc une perte de leur temps et un gaspillage de leur vie (Les Poètes, 129) "Occuperez-vous des châteaux, comme si vous deviez. être immortels?». Ce qui les poussait à se comporter avec brutalité et violence avec les autres )Les Poètes, 130) "Quand vous exercez l'autorité, l'exercezvous comme des tyrans?». Houd leur avait mis une sorte de feuille de route afin qu'ils reviennent à Dieu et vivre dans sa miséricorde (Houd, 52) «O mon peuple! Demandez pardon à votre Seigneur, puis revenez vers Lui. Il vous enverra du ciel une pluie abondante et Il augmentera encore votre force. Ne vous détournez pas [de Lui] en vous rendant coupables ». Il voulait leur 
montrer les richesses dans lesquelles ils vivaient: Dieu leur octroyait beaucoup de grâces, troupeaux et enfants (Les Poètes, 133) «Il vous a comblés de troupeaux et d'enfants », (Les Poètes, 134) "de jardins et de sources ». Ainsi Houd leur avait montré l'importance de rejeter le paganisme et d'adorer Dieu, et comment cela auraient augmenté les bénédictions. Comme le peuple de Noé, ils étaient indifférents à ses appels en insistant sur leur corruption et leur immobilité (Les Poètes, 136) «Ils rétorquèrent : Il nous est indifférent que tu nous exhortes, ou que tu ne nous exhortes pas ». Face à leur refus, Houd craignait un tourment divin contre son peuple (Les Poètes, 135) «Moi, je redoute pour vous le châtiment d'un jour terrible ». Effectivement, un châtiment avait atteint les ('Ad) : leur anéantissement était à travers un vent très froid qui les a arrachés de leurs demeures et les a laissés comme des palmiers déracinés et corrodés (Les Versets Clairement Exposés, 16) «Nous envoyâmes contre eux un vent impétueux durant des jours néfastes, pour leur faire goûter le châtiment de l'ignominie dans la vie de ce monde ... ", (L'Inéluctable, 7) « que Dieu a fait souffler contre eux durant sept nuits et huit jours sans discontinuer. Alors, tu aurais pu voir ces gens couchés à terre comme des troncs de palmiers évidés».

\section{IV.3 Appel de Sâlih}

Dans le Coran, Sâlih était venu après Houd et « Les thamoud sont beaucoup mieux situés dans l'histoire. Déjà sept siècles avant notre ère, le nom apparait dans les documents épigraphiques assyriens »(De Prémare, 1988). En effet, le site archéologique de Al-Hijr, Madâin Sâlih (Madā' in Ṣālih), est inscrit sur la liste du patrimoine mondial (UNESCO : nouveaux biens inscrits (2008)).

Comme tout Envoyé de Dieu, combattre l'idolâtrie était le premier objectif de Sâlih pour guider son peuple vers l'adoration de Dieu. Le peuple de Thamoud était le continuateur de celui des 'Ad : il a hérité son emplacement et ses coutumes en construisant des maisons dans les plaines et sur les montagnes en les sculptant dans les rocs et leurs vestiges témoignent actuellement de cette culture. Les vestiges de Madâin Sâlih ont été découverts dans la cité d'Hégra (Al-Hijr) dans la province d'Al-Hijaz qui est située dans la péninsule arabique, au nord-ouest de l'Arabie saoudite, plus précisément dans le gouvernorat Al- 'Ula de Médine. Il s'agit d'un site antique "composé de quatre ensembles de vestiges : des nécropoles, monumentales et ordinaires, une zone résidentielle largement bâtie, un ensemble de sanctuaires rupestres presque tous situés dans le secteur connu sous le nom de Jabal Ithlib, au nordest, enfin une oasis à laquelle sont associés cent trente puits d'irrigation et des terrains agricoles » (Nehmé, 2005-2006), voir aussi (Nehmé, 2019).

Sâlih invitait les Thamoud à remercier Dieu pour ses bienfaits et à ne pas ruiner la terre de corruption et de péchés (Al-A'râf, 74) «Souvenez-vous 
qu'Il a fait de vous les successeurs des 'Ad et qu'Il vous a solidement établis sur la terre. Dans ses plaines vous aménagez des palais et dans les montagnes vous creusez des maisons. Souvenez-vous donc des bienfaits de Dieu et ne semez pas le mal sur la terre en fauteurs de désordre ». Ce Messager était un maître dévoué à son peuple avant d'être chargé de sa mission (Houd, 62) «...Tu étais, auparavant, un espoir pour nous ». Il avait affirmé qu'il était bien décidé à accomplir sa mission (Houd, 63) et qu'il s'appuyait sur «une preuve évidente ». Il s'adressait à son peuple en attirant leur attention sur la prospérité que Dieu leur avait donnée. Il prêchait en se servant de son éloquence pour raisonner et persuader: (Les Poètes, 146) "Pensez-vous pouvoir jouir indéfiniment de l'ici-bas en toute sécurité ». Son but était d'adoucir leurs cœurs et inciter leur entendement à fonctionner. Il avait commencé par énumérer ce que Dieu leur avait donné comme biens terrestres (Les Poètes, 147) «de jardins et de sources », (Les Poètes ,148) «de cultures et de palmiers élancés », (Les Poètes ,149) «et d'habitations que vous creuserez avec habileté dans les montagnes ?». D’ailleurs, cette description du milieu est confirmée par les découvertes récentes par (Nehmé, cité cidessus)

Il leur avait ordonné, également, de suivre ses conseils et de ne pas aller trop loin dans la désobéissance de Dieu en corrompant la terre (Les Poètes ,150) «Craignez donc Dieu et obéissez-moi », (Les Poètes, 151) «N'obéissez pas aux ordres de ceux qui se livrent à tous les excès », (Les Poètes ,152) « qui répandent la corruption sur la terre et ne s'amendent pas ». Avec son peuple, Sâlih se servait de tous ses arguments et pour le mettre à l'épreuve, il avait eu recours au miracle de la chamelle qui était la concrétisation du principe de la justice divine bafouée par les Thamoud : (Houd, 64) «O mon peuple! Voici la chamelle de Dieu! Elle est un signe pour vous.... Ne lui faites aucun mal ; sinon un châtiment vous saisira promptement ». Cette chamelle miraculeuse prenait la moitié de l'eau et en contre parti, elle nourrissait de son lait tout le peuple. Pourtant, les notables opulents, enflés d'orgueil, refusèrent ce partage : (Al-A'râf, 76) «Les orgueilleux reprirent : Ce à quoi vous croyez, nous le rejetons !», (Al-A'râf, 77) «Ils tranchèrent les jarrets de la chamelle... ». De ce fait, (Al-A'râf, 78) «le cataclysme s'abattit sur eux, et le matin suivant les trouva gisant dans leurs demeures ».

\section{4 Appel de Loth}

Le peuple de Loth occupe une place importante parmi les récits coraniques qui mentionnent clairement la place symbolique de la cité de Sodome (L'Araignée, 35) «Et Nous avons fait en sorte que cette cité reste un signe évident pour les gens qui savent raisonner ». Le châtiment de la cité de Loth, mêlée de fantasme et de réalité, reste un symbole pour tous les temps. En effet, cette cité est située dans la vallée du Jourdain comme 
indiquent les vestiges archéologiques dans la région (Tall el-Hammam) (Collins, 2007 ; Collins \& Latayne, 2013, p.33; Olson, 2014). Selon (Gobillot, 2015) cette cité détient une importance suivant deux dimensions : l'édification spirituelle et l'histoire sacrée.

Loth face à cette cité, tristement célèbre, éprouve durant sa mission, des difficultés énormes car son peuple ne croyait pas aux messagers et les traitait d'imposteurs (Al-Hijr, 74). Loth à qui Dieu avait donné «la sagesse et la science » avait informé son peuple qu'il était le Messager divin sûr et sincère (Les Poètes, 162) «Je suis pour vous un envoyé digne de foi ». Il est présenté par le Coran comme un frère aimant (Les Poètes, 161) "Leur frère Loth leur dit : "Ne craignez-vous pas Dieu ?». Il les avait exhortés à répondre positivement à son appel (Les Poètes, 163) «Craignez donc Dieu et obéissez$m o i$ ». Il les avait avertis de la punition de leur Seigneur s'ils continuaient à vénérer des idoles et commettre des turpitudes choquantes et flagrantes, (AlA'râf, 80) « ... Êtes-vous en train de commettre une turpitude qu'avant vous personne au monde n'a jamais commise?». Mais aucune écoute n'était possible de leur part.

Il est bien connu que Loth avait cru en l'appel d'Abraham, et qu'il l'avait suivi dans sa migration vers la terre bénie, selon (Ibn Kathîr, 2012, p.98-99) : (L'araignée, 26) «Loth crut en lui et lui dit : Moi, je veux émigrer vers mon Seigneur ; Il est, en vérité, le Puissant, le Sage !». Donc, Loth fait partie des continuateurs d'Abraham, qui s'est affirmé comme un modèle monothéiste pour toute l'humanité comme l'affirme (Sayegh, 2020). D'ailleurs, il est important de remarquer que la nouvelle de la punition de la cité de Sodome (L'Araignée, 31) fut annoncée par les anges envoyés auprès d'Abraham qui leur avait demandé d'épargner Loth (Houd, 75). Les anges lui affirment que Loth sera épargné avec sa famille (Al-Hijr, 59) mais à l'exception de (Al-Hijr, 60) « ... sa femme que nous avons destinée à rester parmi les retardataires ».

Loth avait diagnostiqué la corruption de son peuple dans les moindres détails: (Les Poètes, 166) « ... Vraiment, vous êtes un peuple transgresseur !», (Les Fourmis, 55) «Aurez-vous, par concupiscence, commerce avec des hommes plutôt qu'avec des femmes ? Vous êtes vraiment des ignorants!», c'étaient des gens pervertis qui cherchaient à satisfaire pleinement leur instinct (Al-A'râf, 81) «Vous vous approchez des hommes de préférence aux femmes pour assouvir votre désir charnel. Vous êtes un peuple dévoyé ». Loth avait exprimé un dégoût de la cruauté et de la perversion de son peuple (Poètes, 168) «Il dit : Votre façon d'agir m'inspire l'horreur » à causes des leurs actes ignobles et odieux surtout envers ses propres hôtes. En réalité, lorsque Loth avait reçu les anges Envoyés ne savait pas d'où ils venaient, ni ce qu'ils voulaient (Al-Hijr, 62) " ... Vous êtes pour nous des inconnus ! " c'est pourquoi il était dominé par la peur et l'anxiété car si son peuple constatait leur 
présence, ils les attaqueraient, et Loth n'était pas en mesure de les protéger. Ce qui était redouté fut arrivé : la violence des corrompus était grand car ils arrivèrent chez lui, armés de leurs orgueils en espérant commettre la turpitude (Al-Hijr, 67) «Les gens de la ville vinrent tout joyeux s'enquérir des nouveaux arrivants ». Ils avaient traité les hôtes avec effronterie et abjection ce qui avait poussé Loth à crier (Al-Hijr, 68) " ... Ceux-ci sont mes hôtes ; ne me déshonorez pas !», (Al-Hijr, 69) " Craignez Dieu et ne me couvrez pas de honte !». A noter, l'aspect déchaîné qui émanait de leur comportement typiquement animal : ces hommes étaient (Al-Hijr, 72) «ivres et aveugles». Ils voulaient commettre la turpitude avec ses hôtes qui n'étaient que les anges sous forme humaine; alors, Loth les grondait pour leur horreur, en se demandant s'il y avait un homme droit parmi eux qui pouvait les empêcher de commettre cette iniquité (Houd, 78) « ... Il leur dit : O mon peuple ! Voici mes filles! Elles seraient plus pures pour vous! Craignez Dieu et ne m'outragez pas dans mes hôtes! N'y a-t-il pas parmi vous un seul homme droit ? ». Mais ils rétorquèrent (Houd, 79) «Tu sais parfaitement que nous n'avons rien à voir avec tes filles, et tu sais bien ce que nous voulons ! ». Loth était accablé par le désespoir en étant incapable de stopper leur fourvoiement : son chagrin était énorme, il se sentait désarmé devant leur immoralité en cherchant un endroit pour s'abriter avec ses hôtes (Houd, 80). Effectivement, Sodome faisait partie des cités que le Coran appelait « les cités renversées », (almū' tafikāt), celles qui commirent des péchés graves.

Après toute cette perversion, Dieu décide de mettre fin à leur abjection : (Al-Hijr, 73) «Le cri les saisit à l'aube » et leur citée fut détruite. Dans ce contexte, (Gobillot, 2015) s'interroge sur les causes physiques de sa disparition «tremblement de terre et éruption volcanique » et sur la localisation de son emplacement « entre "Via Nova Traiana" (une ancienne route romaine) et chemin caravanier de la Mer Morte ».

\section{IV.5 Appel de Chu'aïb}

Après les quatre précédentes cités maudites de Noé, Loth, Houd et Saleh, le peuple de Chu'aïb avait les vices de ses prédécesseurs et se distinguait par la fraude en toutes sortes. Comme les autres Messagers, Chu'aïb était venu portant un message de Dieu à son peuple suite à leur mauvaise conduite (Les Poètes, 178) "Je suis pour vous un envoyé digne de foi ». Homme bien connu de son peuple, ce Messager ne voulait que sauver les siens de leur corruption. Il ne désirait ni le pouvoir ni les récompenses : (Les Poètes, 180) " Je ne vous demande en échange aucun salaire... ". Il les invitait à la reforme pour sortir des méandres de l'escroquerie selon des principes simples et applicables.

Mais quelle est cette preuve patente parvenue du Seigneur (Al-A'râf, 85) que Chu'aïb avait annoncée à son peuple ? Effectivement, il s'agit d'une 
reforme basée sur deux socles : le renoncement au paganisme et à la corruption: Chu'aỉb partait d'un diagnostic solide de la réalité de la décomposition de la société et proposa des solutions qui permettaient à chacun de vivre en sécurité.

Il avait défini les fondements du bien de son peuple et de tous les humains à travers un triangle, que l'on pourrait appeler " le triangle de la gouvernance sociale". Comme on peut le remarquer dans le verset (Al-A'râf, 85) « ... O mon peuple! Adorez Dieu! Il n'y a pas, pour vous, d'autre dieu que Lui. Une preuve patente vous est déjà parvenue de votre Seigneur. Donnez bonne mesure et bon poids. Ne causez pas de tort aux hommes dans leurs biens. Ne semez pas la corruption sur la terre après qu'elle aura été amendée. Ce sera un bien pour vous si vous êtes croyants ». Dans ce triangle, il y a un ordre et deux impératifs négatifs, et la figure suivante reflète clairement cette idée.

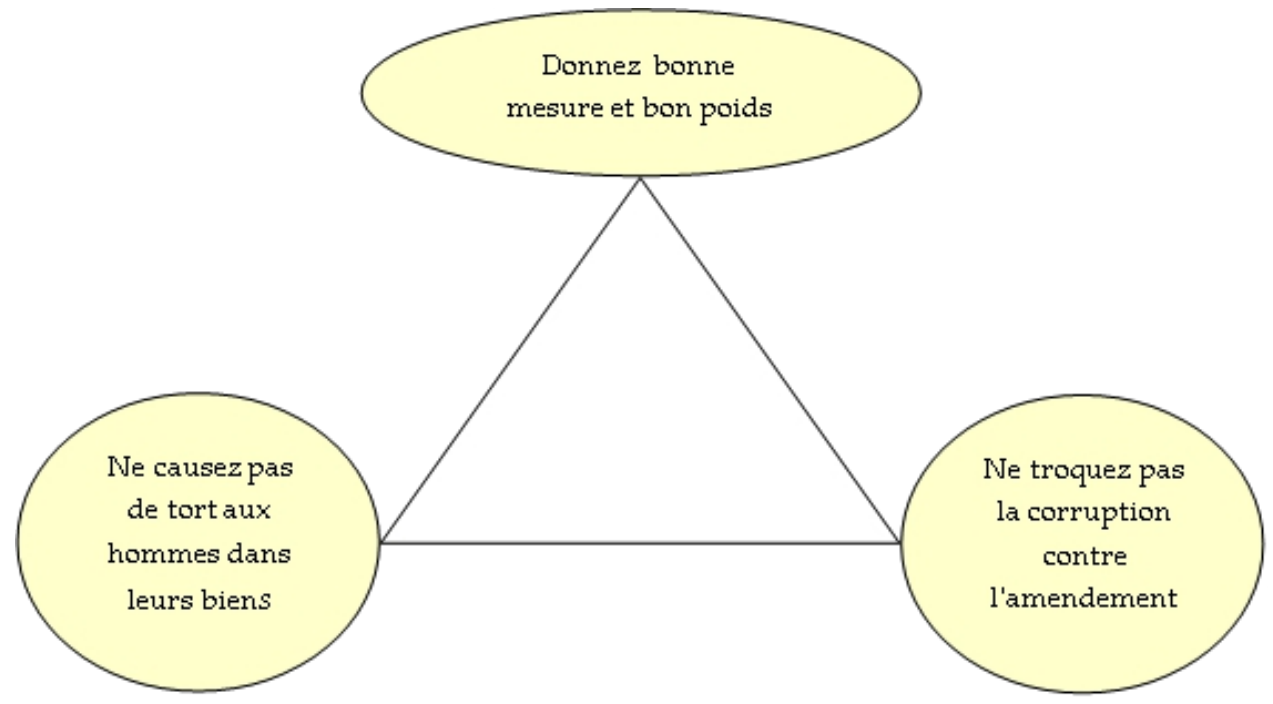

Les appels de Chu'aïb au respect du poids et de la mesure ne font que pousser « les gens d'Al Aïka » à pratiquer plus de fraudes en arguant qu'ils avaient le droit de commercer comme bon leur semblait en l'accusant de tromperie et de déviation (Houd, 87). Les principes annoncés par le Messager leur semblaient comme une ingérence dans leurs affaires et une atteinte à leur liberté d'exercer le commerce.

Après avoir épuisé tous les moyens de conviction, Chu'aïb avait eu l'audace de prévenir son peuple du risque de subir des maux : (Houd, 89) «... pareils à ceux qui ont frappé le peuple de Noé, ou le peuple de Houd, ou le peuple de Sâlih! Et aussi le peuple de Loth, qui n'est pas très éloigné de vous !». Mais les notables, premiers bénéficiaires de l'état de corruption de leur cité, étaient à la tête des opposants farouches qui menaçaient Chu'aïb de 
lapidation et d'expulsion (Houd, 91) « ... Et si ce n'était par égard pour ton clan, nous t'aurions certainement lapidé. De fait, tu ne possèdes aucun pouvoir contre nous! ». Chu'aïb avait répondu par une logique importante : comment peuvent-ils craindre son clan alors qu'ils bafouaient les appels de Dieu?

Les arguments factuels et les raisonnements de Chu'aïb étaient restés sans effet. Ainsi, désarmé devant leur ténacité et leur arrogance, il s'adressait à son peuple dans une menace ultime : (Houd, 93) «O mon peuple! Agissez selon vos possibilités, j'agirai aussi! Bientôt vous saurez sur qui tombera un châtiment ignominieux, et qui est menteur. Veillez donc; je veillerai avec vous ». Enfin, Dieu leur imposa un grand cataclysme (Houd, 94) « ... Le cri saisit ceux qui avaient été iniques et, le matin suivant, ils gisaient dans leurs demeures».

\section{Conclusion}

Pris dans leur totalité thématique, les versets du Coran ont permis de concevoir une vision globale des cités maudites caractérisées par leur aveuglement et leur arrogance (Al-Qasas, 58) «Combien avons-Nous détruit de cités dont les habitants jouissaient insolemment de leur abondance! Voici leurs demeures, restées presque inhabitées après leur mort. Et c'est Nous qui en avons hérité ». Les fouilles archéologiques d'Al-Hijr en Arabie saoudite, et de Tall El-Hamam en Jordanie, ont démontré l'existence historique des cités (Madā' in Șāliḥ) et Sodome, qui ne sont plus des fantômes qui hantent l'imaginaire collectif. Leurs architectures témoignent d'une prospérité certaine qui incite à chercher les raisons de leur anéantissement dramatique et définitif.

Des faits qui incitent à chercher les raisons de leur destruction définitive. En réalité, leurs maux, dans leur diversité, révèlent les causes de l'anéantissement des sociétés humaines à travers le temps: injustice, immobilisme, dévouement inconditionné aux coutumes anciennes, fanatisme, corruption, discrimination entre grands riches et humbles, acharnement pour les gains illicites et les fraudes, déviations et turpitudes. Tout cela dans une résistance aux appels à la réforme lancée par les Envoyés divins. A cet effet, les cités n'ont pas hésité à les terroriser en les menaçant de lapidation et de mort. Tout cela était orchestré par une classe composée de notables opulents et criminels qui détenaient tous les pouvoirs, sociaux, économiques et religieux sous prétexte d'appartenir aux grands riches seuls capables de diriger (La vache, 11) "Lorsqu'on leur dit: Ne semez pas la corruption sur la terre ", ils répondent: " Nous ne sommes que des réformateurs!».

Les expériences respectives des Messagers révèlent que le processus de la destruction n'est pas le résultat d'une décision arbitraire de la part de Dieu, mais au contraire le résultat d'un débat entre les cités et leurs Envoyés 
où chaque parti avait montré ce qu'il possède comme arguments. Dans ce domaine, les Messagers qui affectionnaient leurs peuples, ont déployé, en vain, toutes leurs capacités pour les convaincre. Ils faisaient partie intégrante de ces cités et usaient même de leur notoriété pour influencer positivement les membres de leurs communautés. Loin de tout intérêt privé, chaque Messager proclamait son opinion en visant l'intérêt exclusif de son peuple. A cet égard, il convient de préciser que "si seulement le fait de servir les autres était poussé par l'égoïsme, quel serait alors la valeur inhérente à cette action!» selon (Kamel, 1954, p.44). En fait, chaque Envoyé avait comme mission de proclamer le message en toute clarté en s'appuyant sur un dialogue rationnel qui motive l'esprit des gens conformément à la loi divine selon laquelle l'argument précède la destruction ou le châtiment. L'enchaînement des cités maudites à leurs concepts et à leurs traditions païennes ancrées dans leur ordre moral, les a empêchées de répondre aux appels des Messagers. La menace d'expulsion ou de meurtre à leur égard était une expression de cet attachement à leur immobilité.

Les premières tentatives réformatrices étaient toujours guidées par la douceur des propos où l'accent affectif dominait pour faire appel aux cœurs et aux esprits. Souvent le messager essayait de partir des constats réels, comme les richesses et les bienfaits naturels, pour encourager son peuple à se rapprocher de Dieu et abandonner leurs idoles. Les Messagers adoptaient une méthode qui exhorte l'entendement à faire la distinction entre le bien et le mal en suivant une ligne ascendante : leur objectif était de prévaloir la raison sur les penchants naturels de l'homme. A cet effet, les Messagers ont usé d'un style argumentatif accessible aux masses dans le but de montrer l'importance de la logique et la force de la persuasion, une occasion de confirmer le rôle de la logique dans le domaine de la foi. Un signe aussi qu'aucune réforme n'est possible qu'accompagnée de l'adhésion de la société qui l'adopte par conviction et non pas à travers la contrainte. L'homme s'affirme, ainsi, comme l'auteur de son propre sort qui se dessine d'après son acte (L'Étoile, 39) « et que l'homme n'aura que le fruit de ses efforts ».

La personnalité du prophète selon le Coran à travers son récit est la concrétisation de ce principe d'intégrité qui triomphe de l'injustice, comme l'illustre la sourate (Joseph) consacrée au récit édifiant de Joseph (Sayegh, M, 2019). Cette idée est illustrée surtout par l'appel à la droiture dans les pratiques économiques et sociales qui met l'accent sur l'importance de l'équité qui se révèle comme le contraire de l'iniquité, cause principale des malheurs des peuples : fausser la mesure et le poids est un acte qui ne se limite pas aux ventes et aux achats, mais il s'étend à tous les domaines où s'exerce la volonté humaine.

En effet, quand chaque personne accomplit correctement son rôle selon les lois de la conscience en respectant le principe de « la bonne mesure et le 
bon poids » adopté comme une règle de conduite, alors la justice, l'équilibre et la paix s'instaurent dans la société humaine. L'honnêteté dans les mesures ne fait qu'aboutir à une meilleure perception des capacités de chacun et de son rôle au sein de la communauté. Cela conduirait à la prospérité de tous et assurerait le bien-être humain. Interdire la corruption après la réforme finit par l'accumulation des bienfaits ce qui aboutit à l'évolution des sociétés.

Cette idée d'intégrité se reflète aussi dans la décision même du châtiment divin subi par les cités maudites : aucune destruction n'est faite sans un avertissement préalable par un Messager dans un long processus d'argumentations et preuves logiques et expérimentales qui épuisent toutes les possibilités de convaincre (Les Poètes, 208,209) «Nous n'avons détruit aucune cité sans qu'elle ait reçu des avertisseurs », "et un rappel. Nous $n^{\prime}$ avons pas agi injustement ». À partir de cette vision, et compte tenu de la relation dialectique entre la relation de cause à effet, la corruption conduit à la destruction. Les Messagers ont épuisé toutes leurs forces en prouvant énormément de patience et de tolérance face aux insultes méprisantes de leurs peuples qui les ont accusés d'imposture, de folie et de lâcheté. Alors quand ils sont devenus incapables d'apporter une sortie à leurs peuples, c'était l'intervention divine directe qui a détruit, par déluge, le peuple de Noé, par un vent mugissant et impétueux, le peuple de Houd, par le cri, les peuple de Sâlih et Chu'aïb, par des pierres d'argile, le peuple de Loth.

La destruction ne serait pas arrivée si les cités n'avaient pas été submergées complètement par leur injustice qui s'exprimait par leur corruption et leur débauche (Le Pèlerinage, 45) «Combien de cités n'avonsNous pas détruites parce qu'elles étaient iniques. Elles sont maintenant désertes et ruinées. Que de puits sont abandonnés et de palais écroulés!»; (Houd, 100,101) «... dont certaines sont encore debout tandis que d'autres ont été moissonnées », et «Nous ne les avons pas lésées, mais elles se sont fait tort à elles-mêmes... ». Cela confirme la loi divine qui est basée sur un principe logique selon lequel la preuve précède toujours la destruction : c'est une façon constructive de pousser les gens à se réveiller de leur léthargie en réfléchissant à tout ce qui les entoure comme révèle (Fadlallah, 1998, p.17, p.135), et ce que confirment les versets (Al-Talâq, 8-9) "Que de cités ont été rebelles à l'ordre de leur Seigneur et de Ses envoyés! Nous avons tenu pour elles un compte sévère, et Nous leur avons infligé un rude châtiment».

\section{References :}

1. Abd Al Haye, M. Y. (2016). Matériaux et entrées dans le dictionnaire historique (éd. 1). Le Caire, Egypte: Dar Al-Kotob.

2. Alam, Y. M. (2016). les verbes, les racines et structures en langue arabe. Journal de la Faculté de langue arabe, Université Al-Azhar, Partie III,(35), 1403-1443. 
3. Bell, D. (1989). Jorge Luis Borges: Jeux avec le temps et avec l'infini. Érudit/ Revues/ Nuit blanche, magazine littérairel Visions sudaméricaines(Numéro 38, décembre 1989, janvier-février 1990), 3441. Retrieved from https://id.erudit.org/iderudit/19736ac

4. Collins, S. (2007). A Response to Bryant G. Wood Critique of Collins' Northern Sodom Theory. Biblical Research Bulletin, 7(7), 1-36.

5. Collins, S. \& Latayne, C. S. (2013). Discovering the City of Sodom: The Fascinating, True Account of the Discovery of the Old Testament's Most Infamous City. New York: Howard Books.

6. De Prémare, A.L. (1988). Le thème des peuples anéantis dans quelques textes islamiques primitifs. Une vision de l'histoire. Revue des mondes musulmans et de la Méditerranée(48-49), 11-21.

7. De Prémare, A.L. (2006). Le Coran, enquête sur un livre sacré. 1-7. doi:https://www.lhistoire.fr/parution/collections-30.

8. Déclais, J.-L. (2015). Une écriture dont les textes sont interprétés, Le cas de la sourate VII (al-A 'rāf). Mélanges de l'Institut dominicain d'études orientales (MIDÉO),(31), 131-179.

9. Electronic Qur'an , www.E-Quran.com (2009).

10. Elster, J. (2010). Obscurantisme dur et obscurantisme mou dans les sciences humaines et sociales (Diogène ). (1 ( $\left.\left.n^{\circ} 229-230\right)\right), 231-247$. doi:10.3917/dio.229.0231

11. Fadlallah, M. H. (1998). Recueil des séminaires (éd. 4, Vol. 1). Haret Hreik, Lebanon: Dâr al-Malak.

12. Fadlallah, M. H. (1998). Interprétation inspirée du Coran (éd. 2, Vol. 14). Haret Hreik, Liban: Dâr Al-Malak,.

13. Fadlallah, M. H. (1998). Interprétation inspirée du Coran (éd. 2, Vol. 17). Haret Hreik, Liban: Dâr Al-Malak.

14. Fadlallah, M. H. (1998). Interprétation inspirée du Coran, Haret Hreik, Liban (éd. 2, Vol. 19). Dâr Al-Malak.

15. Fadlallah, M. H. (1998). Interprétation inspirée du Coran (éd. 2, Vol. 9). Haret Hreik, Liban: Dâr Al-Malak.

16. Frégosi, F. (2002). Laïcité et religions : Le coran un texte politique ? Revue des Deux Mondes, 68-76.

17. Gnos, E., Hofmann, B. A., Halawani, M., Tarabulsi, Y., Hakeem, M., Al Shanti, M., . . . Ramseyer, K. (2013). The Wabar impact craters, Saudi Arabia, revisited. Meteoritics \& Planetary Science, 48(10), 2000-2014. doi:10.1111/maps.12218

18. Gobillot, G. (2015). Histoire et géographie sacrées dans le CoranL'exemple de Sodome . Mélanges de l'Institut dominicain d'études orientales (MIDÉO), 1-54.

19. Gouguenheim, S. (2008). Aristote au Mont-Saint Michel. Paris: Seuil. 
20. Ibn Kathîr, I. (2012). L'exégèse du Coran, Dâr Al -kotob Al-ilmiyah (éd. 8, Vol. 3). Betrouth, Liban: Dâr Al-kotob Al-ilmiyah.

21. Kamel, M. H. (1954, 1997). Cité Maudite. Le Caire, Égypte: Dâr alkotob. Récupéré sur Association Égyptienne Générale des livres

22. Nehmé, L. (2005-2006). Inscriptions nabatéennes vues et revues à Madā' in Șālih. Arabia,(3), 179-225, fig. 117-155, pp. 345-356.

23. Nehmé, L. (2019). Report on the 2019 Season of the Madâ'in Sâlih. Archaeological Project. Edited by Laïla Nehmé.

24. Olson, C. (2014). Which Site Is Sodom? A Comparison of Bab edhDhra and Tall el-Hammam. Biblical Research Bulletin, 14(1), 1-17.

25. Prescott, J. \& Robertson, G. (2004). Luminescence dating of the Wabar meteorite craters, Saudi Arabia,. Journal Of Geophysical Research, 109, 1-8. doi:10.1029/2003JE002136

26. Renan, E. (1992, 1857,1886). Études d'histoire religieuse. Paris: Gallimard.

27. Richard, A. J. \& Gouri, K. B. (1996). Statistics:Principles and methods (éd. 3). New York. Chichester. Brisbane. Toronto. Singapore: John Wiley and Sons,Inc.

28. Sayegh, M. (2019). La sourate Joseph : Approche mytho-critique. Jordan Journal of Modern Languages and Literatures, 11(2), 111-126.

29. Sayegh, M. (2020). Abraham dans le texte coranique: Itinéraire vers la convergence universelle. European Scientific Journal (ESJ), 16(17), à paraître en juin 2020.

30. Shahrur, M. (2018). L'état et la société : destruction des (al-qura : les cités) et prospérité des villes (al-modon). Beyrouth, Liban: Dar Al Saqi.

31. Taharraoui, Y. (2015). La figure de Noé entre le Coran et les isrā'īliyyāt. Revue de l'histoire de religions(4), 645-682.

32. https: $/ /$ whc.unesco.org/fr/nouveauxbiens/date $=2008 \&$ mode $=$ list

33. https://www.larousse.fr/dictionnaires/francais/idol\%C3\%A2trie/4146 $\underline{3}$ 